\title{
Ultrasound screening for hepatocellular carcinoma in patients with advanced liver fibrosis. An overview.
}

\author{
Mirela Dănilă, Ioan Sporea
}

Gastroenterology and Hepatology Department, University of Medicine and Pharmacy "Victor Babeș", WFUMB Center of Excellence, Timişoara, Romania

\section{Abstract}

Liver cirrhosis is the major risk factor for hepatocellular carcinoma (HCC). All etiologic forms of liver cirrhosis may be complicated by HCC but the risk is higher in patients with hepatitis B or C viral infection. Patients at high risk for developing HCC should be included in surveillance programs. Groups at high risk are: all cirrhotic patients; non-cirrhotic patients with chronic hepatitis $\mathrm{C}$ and advanced liver fibrosis (F3), non-cirrhotic HBV carriers with active hepatitis. Current guidelines issued by AASLD and EASL recommend surveillance among high-risk patients by ultrasound (with or without alpha-fetoprotein) every 6 months. The aim of ultrasound screening and surveillance is to detect HCC at an early stage, when it is amenable to curative therapy, to reduce mortality. For good results, ultrasound examination must be done by highly experienced personnel, with extensive experience in the field of hepatic ultrasound.
\end{abstract}

Keywords: liver cirrhosis, hepatocellular carcinoma, ultrasound, screening.

Hepatocellular carcinoma (HCC) is the most frequent primary liver cancer occurring frequently in patients with liver cirrhosis and in patients with chronic liver disease and advanced fibrosis.

The incidence of HCC is rising in many countries (especially in developing countries), being responsible for more than 500,000 deaths per year. In Romania, according to data provided by GLOBOCAN, in 2008 the incidence of liver cancer for both genders was 5.3/100,000 $(8.1 / 100,000$ in men and $3 / 100,000$ in female patients) [1].

Cohort studies indicated that HCC is currently the major cause of liver-related death in patients with compensated cirrhosis. Hepatitis $\mathrm{C}$ virus (HCV) and hepatitis $\mathrm{B}$ infection (HBV) are associated with the highest $\mathrm{HCC}$ incidence in persons with cirrhosis.

Treatments modalities (curative treatment) in patients with HCC are frequently outdated at the time of diagno-

Received 24.02.2014 Accepted 15.03.2014

Med Ultrason

2014, Vol. 16, No 2, 139-144

Corresponding author: Mirela Dănilă

73/9 Martir Petru Domăşneanu street

300351Timişoara, Romania

E-mail:mireladanila@gmail.com sis, due to advanced disease. Prognosis of HCC is determined by tumor stage at diagnosis and also by the stage of liver disease.

Because HCC occurs most often on a background of chronic liver disease, it is important to screen this class of patients for early detection. Ultrasonography (US) is usually used for HCC screening due to its cost-effectiveness and non-invasiveness. Contrast-enhanced ultrasound (CEUS), contrast enhanced computer tomography (CE$\mathrm{CT}$ ) or contrast enhanced magnetic resonance imaging (CE-MRI) are the methods used to certify the diagnosis of HCC.

\section{Target populations for screening program}

Cirrhosis of any etiology is the major risk factor for HCC; in approximately $90 \%$ of cases, hepatocellular carcinoma occurs in patients with liver cirrhosis [2].

The risk of HCC varies, according to the etiology of liver cirrhosis, ranging between $1.5-4.5 \%$ per year in $\mathrm{HCV}$ related cirrhosis [3] and 2.2-4.3\% per year in HBV related cirrhosis [4].

Also, the incidence of HCC in viral C liver cirrhosis has been reported differently according to the geographi- 
cal region. In a retrospective cohort study, the annual $\mathrm{HCC}$ incidence was 1.4\% in European cirrhotic HCV patients [5]. Degos et al [6] reported HCC incidence rates of $13.4 \%$, after five years follow-up in a French cohort of patients with compensated cirrhosis. In the study performed by Lok et al [7] in the United States, the HCC incidence was higher in $\mathrm{HCV}$ patients with cirrhosis, than in those with bridging fibrosis (annual $\mathrm{HCC}$ incidence rates: $1.45 \%$ vs $0.8 \%, p=0.08$ ).

Infection with $\boldsymbol{H B V}$ and $\boldsymbol{H C V}$ are the major risk factors for the development of $\mathrm{HCC}$.

For patients with chronic hepatitis $C$ infection, $\mathrm{HCC}$ surveillance is recommended in any patient who has developed cirrhosis. Some experts also recommend screening for $\mathrm{HCC}$ in $\mathrm{HCV}$-infected patients who have advanced fibrosis, but no cirrhosis (METAVIR stage 3). $\mathrm{HCC}$ screening is not recommended in HCV-infected patients with no advanced fibrosis or cirrhosis.

The risk for HCC decreases substantially in patients with cirrhosis who obtain a sustained virologic response (SVR) with therapy for hepatitis $\mathrm{C}$, but this risk is not totally eliminated. This category of patients must also be included in the screening program for early detection of HCC.

Chronic hepatitis B virus ( $\mathrm{HBV}$ ) infection is estimated to be the cause of $55-60 \%$ of HCCs in the world, up to $40 \%$ of HBV-related HCC occurring in persons who do not have cirrhosis [8].

Many studies have reported relatively a high incidence of HCC in HBV carriers [9]. In a Taiwanese study, the annual incidence rate of HCC was $0.5 \%$ [10], but another study from North America indicated that HCC incidence in HBV carriers varies considerably, with annual HCC incidence rates ranging between $0.06 \%$ and $0.46 \%[11,12]$.

In hepatitis B viral infection, high transaminases, $\mathrm{HBeAg}$ positive status, and high serum HBV DNA levels were risk factors for developing HCC [13].

On the other hand, any patient with liver cirrhosis can develop HCC, including patients with cirrhosis from non-viral causes, such as alcoholic cirrhosis, non-alcoholic fatty liver disease (NAFLD), autoimmune hepatitis, and other rare causes of cirrhosis like hemochromatosis, Wilson's disease or alpha-1-antitrypsin deficiency.

In the later years, liver cirrhosis due to NAFLD is recognized as an important risk factor for HCC. The incidence of NAFLD/NASH (non-alcoholic steatohepatitis) is rising in many countries, in conjunction with obesity, diabetes or dyslipidemia. In a systematic review performed by Wite et al [14], an association between NAFLD/NASH and an increased risk for $\mathrm{HCC}$ has been found, but only in patients with cirrhosis.
All HCC Guidelines (from the American Association for the Study of Liver Disease - AASLD and the European Association for the Study of the Liver - EASL) $[15,16]$ recommend the screening for patients at high risk for developing HCC. These categories of patients are:

1. Cirrhotic patients, Child-Pugh stage A and B;

2. Cirrhotic patients, Child-Pugh stage $C$ awaiting liver transplantation;

3. Non-cirrhotic HBV carriers, with active hepatitis or a family history of HCC;

4. Non-cirrhotic patients with chronic hepatitis $\mathrm{C}$ and advanced liver fibrosis F3.

For patients with liver cirrhosis Child Pugh stage C, who are not candidates for liver transplantation, surveillance is not recommended, since life expectancy is due to the stage of the liver disease.

\section{US for HCC surveillance}

Current guidelines from AASLD and EASL recommend surveillance among high-risk patients by ultrasound [with or without alpha-fetoprotein (AFP)] every 6 months. The aim of US screening and surveillance is to detect HCC at an early stage, when it is amenable to curative therapy in order to reduce mortality. For a good result, US should be performed by experienced personnel.

US is a noninvasive method, without any risk for the patients, well accepted by them and also inexpensive, thus making US a repetitive examination in patients with liver cirrhosis. But US detection of HCC on a cirrhotic background is a challenging issue.

Liver cirrhosis is characterized by fibrous septa and regenerative nodules. These hepatic features make the evaluation of liver texture sometimes difficult. The sensitivity of US for HCC detection is also significantly associated with patients characteristics - difficult in obese patients, with poor hepatic window.

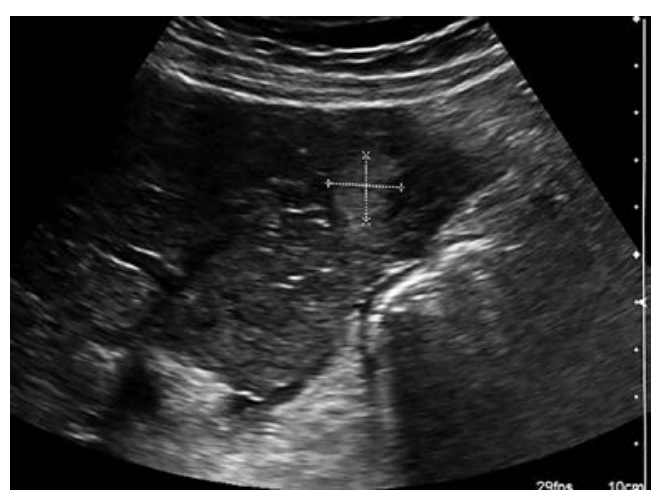

Fig 1. Hyperechoic hepatocellular carcinoma - aspect in standard US 


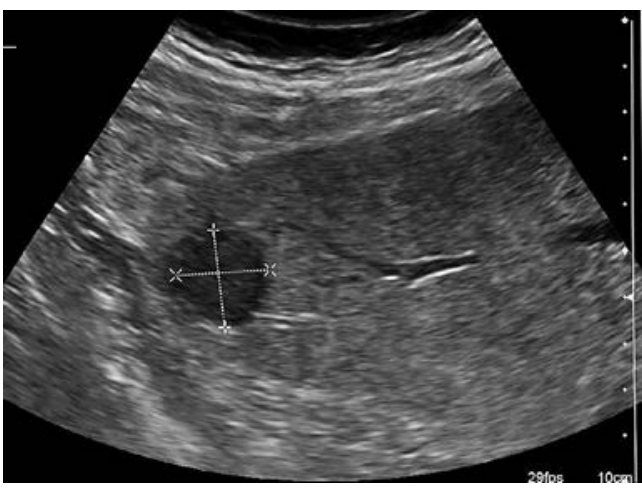

Fig 2. Hypoechoic hepatocellular carcinoma - aspect in standard US

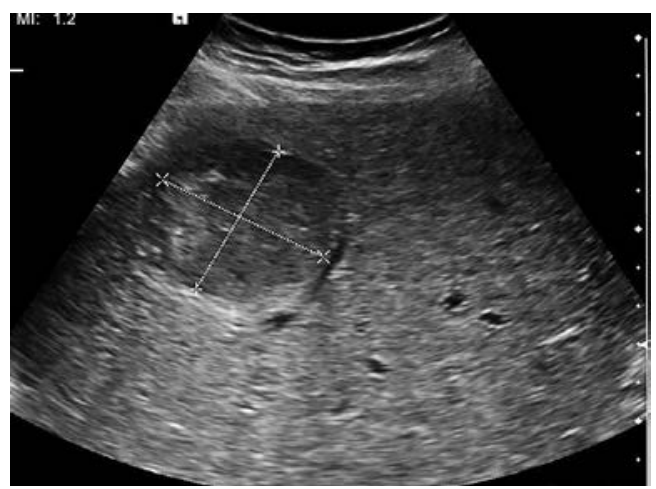

Fig 3. Inhomogeneous hepatocellular carcinoma aspect in standard US
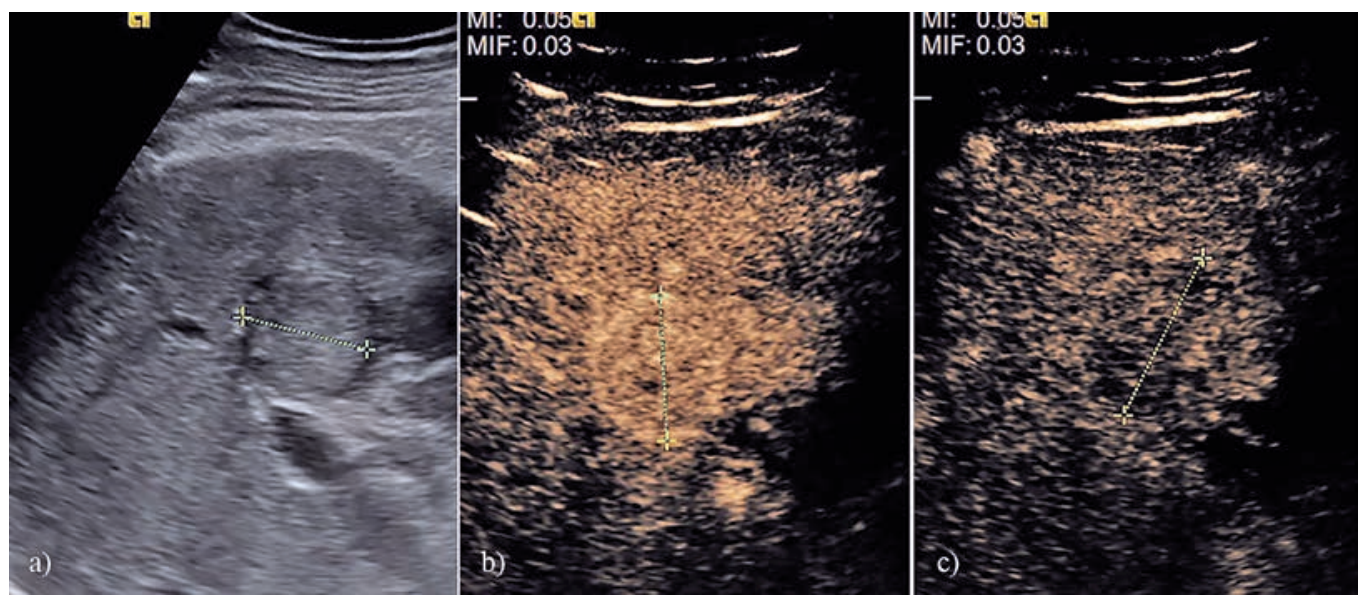

Fig 4. CEUS examination in a patient with hepatocellular carcinoma (HCC): a) - HCC - aspect in standard ultrasound; b) - CEUS examination - arterial phase with hyperenhancement pattern; c) - CEUS examination - late phase with wash out

Other factors that influence the accuracy of US in the evaluation of liver structure are: technical performance of the US machine (US resolution has been much improved in the latest years), and also the experience of the examiner. Examination of a cirrhotic liver for detection of HCC must be done by highly experienced ultrasonographists, level 2 or 3 according to the EFSUMB and SRUMB classification of Level of Practice for US (www.efsumb.org or www.srumb. ro).

The US appearance of HCC varies: it may be hyperechoic or hypoechoic (fig 1, fig 2), or it may appear as a "target" lesion. Sometimes, a small hyperechoic HCC may be confused with hemangioma. Large HCCs are more frequent inhomogeneous (fig 3 ) and sometimes associated with portal vein thrombosis.

Because of the increased risk of developing cancer, a liver mass in a cirrhotic patient strongly suggests the possibility of a HCC, but the final diagnosis of $\mathrm{HCC}$ is based on imaging methods with contrast media. In the later years, CEUS performed immediately after standard US examination allows the diagnosis of $\mathrm{HCC}$ in the same session [17-19] (fig 4).

Sensitivity and specificity of US for early detection of HCC was evaluated in numerous studies, as well as the superiority of screening versus non-screening strategies in patients with risk for HCC.

The optimal interval for ultrasonographic surveillance for early detection of HCC is not known. Based on the median doubling time of HCC [20] (range between 80 and 117 days), the recommended interval between HCC surveillance tests is $\mathbf{6}$ months.

In one placebo-controlled randomized study [21], which included 19,000 HBV-infected patients, it was shown that HCC surveillance with both abdominal ultrasound and serum AFP repeated at 6 month intervals, 

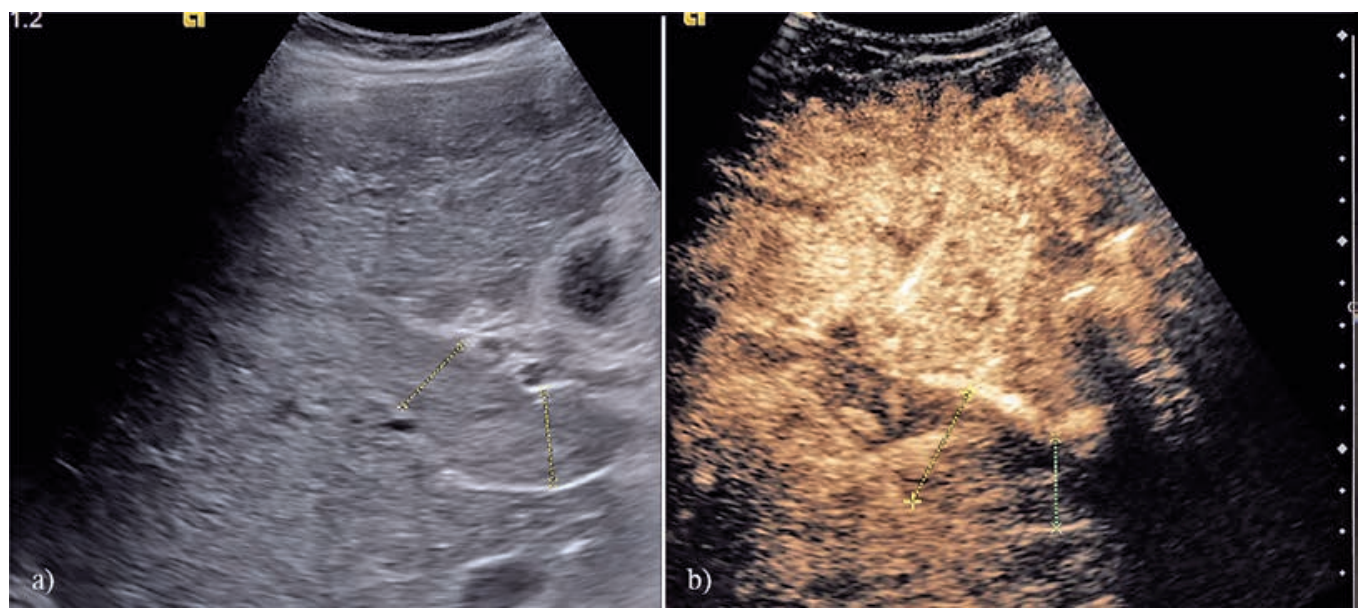

Fig 5. Ultrasound in portal vein thrombosis (PVT): a) - PVT - aspect in standard ultrasound; b) - PVT - CEUS aspect with arterial enhancement in malign PVT.

resulted in a $37 \%$ reduction in HCC-related mortality. According to the meta-analysis by Singal et al [22], US sensitivities for detecting early-HCC may be improved by US at 6 month intervals, compared to surveillance intervals between 6-12 month (sensitivities: $70 \%$ vs 50\%, $p=0.001)$. A recent randomized control trial [23] investigated whether a 3-mo interval of US surveillance was more effective than a 6-mo interval and no differences in detection rates of small HCC, eligible for curative treatment, were observed between the two randomized groups.

A recent meta-analysis [24] has shown that US can identify subclinical HCCs with a sensitivity of $94 \%$ and $90 \%$ specificity, but US was less effective for detecting early HCC (Barcelona Clinic Liver Cancer stage A), with a sensitivity of $63 \%$. In the same study, combination of US and serum AFP assessment slightly increased (6\%) the sensitivity of surveillance, but almost doubled the cost for each small HCC detected, due to a high number of false positives results.

In a study [25] that evaluated the effectiveness of a surveillance program using ultrasound and AFP every 6 to 12 months among patients with cirrhosis, in a realworld clinical setting, the sensitivity of ultrasound alone for early-stage tumors was only $32 \%$. The sensitivity was significantly increased to $63 \%(p=0.008)$, when used in combination with AFP.

Until now, there are no official recommendations regarding the use of CE-CT or CE-MRI for HCC screening (due to the high cost), but when US visualization of the liver is inadequate, mostly in patients with liver cirrhosis, CE-CT or CE-MRI are frequently used in clinical practice for HCC screening.
All studies demonstrated that survival was significantly improved among patients diagnosed with HCC, who had undergone prior screening. This was primarily due to curative therapy in patients with earlier stage HCC and less severe liver disease.

\section{AFP for screening in $\mathrm{HCC}$}

Worldwide, AFP is the most frequently used biomarker for HCC, but not all HCC cells secrete AFP into the circulation. Also, serum AFP levels may be elevated in patients with chronic liver disease in the absence of HCC. In one systematic review, using the usual cut-off point of $20 \mathrm{ng} / \mathrm{mL}$, sensitivities and specificities for detecting all stages of $\mathrm{HCC}$ were $41 \%-65 \%$ and $80 \%-94 \%$, respectively [26]

The use of serum AFP as a surveillance test has a poor sensitivity for early HCC, since only a small proportion $(10-20 \%)$ of early cancers, are associated with elevated AFP serum levels [27,28].

A recent cost-effectiveness analysis found that the combination of ultrasound and AFP was the preferred strategy, when the sensitivity of ultrasound fell below $65 \%$ [25].

Thus, in clinical practice, US will be used for screening for $\mathrm{HCC}$ in specific categories of patients. For good results, only trained physicians, with extensive experience in the field of hepatic US, must perform this procedure (level 2 or 3 according to the EFSUMB and SRUMB classification). Also mandatory is the use of high tech US machines, preferable able to perform CEUS. In patients with a good acoustic window, this method is usually highly accurate for the diagnosis of HCC (and also for staging the tumor, concerning a possible portal thrombo- 
sis - fig 5) [29]. In cases that are not suitable for ultrasound examination, CE-CT or CE-MRI are the alternatives for screening.

\section{Conclusions}

All patients with advanced liver fibrosis and risk for HCC must undergo ultrasound evaluation every 6 months. Extensive US experience and good US machines are essential for a correct diagnosis.

Conflict of interest: none of the authors had any conflict of interest.

\section{References}

1. Ferlay J, Shin HR, Bray F, Forman D, Mathers C, Parkin DM. GLOBOCAN 2008 v1.2, Cancer Incidence and Mortality Worldwide: IARC CancerBase No. 10, IARC, Lyon, 2010, http://globocan.iarc.fr. Accessed on 07/04/2012.

2. El-Serag HB. Hepatocellular carcinoma. N Engl J Med 2011;365:1118-1127.

3. Fattovich G, Stroffolini T, Zagni I, Donato F. Hepatocellular carcinoma in cirrhosis: incidence and risk factor. Gastroenterology 2004; 127: S35-S55.

4. Gomez EV, Rodriguez YS, Bertot LC, et al. The natural history of compensated HCV-related cirrhosis: a prospective long-term study. J Hepatol 2013; 58: 434-444.

5. Fattovich G, Pantalena M, Zagni I, Realdi G, Schalm SW, Christensen E; European Concerted Action on Viral Hepatitis (EUROHEP). Effect of hepatitis B and $\mathrm{C}$ virus infections on the natural history of compensated cirrhosis: a cohort study of 297 patients. Am J Gastroenterol. 2002; 97: 2886-2895.

6. Degos F, Christidis C, Ganne-Carrie N, et al. Hepatitis C virus related cirrhosis: time to occurrence of hepatocellular carcinoma and death. Gut 2000; 47: 131-136.

7. Lok AS, Seeff LB, Morgan TR, et al; HALT-C Trial Group. Incidence of hepatocellular carcinoma and associated risk factors in hepatitis C-related advanced liver disease. Gastroenterolog 2009; 136: 138-148.

8. Lok AS. Hepatitis B: liver fibrosis and hepatocellular carcinoma. Gastroenterol Clin Biol 2009; 33: 911-915.

9. De Franchis R, Meucci G, Vecchi M, et al. The natural history of asymptomatic hepatitis B surface antigen carriers. Ann Intern Med 1993; 118: 191-194.

10. Beasley RP, Hwang LY, Lin CC, Chien CS. Hepatocellular carcinoma and hepatitis B virus. A prospective study of 22 707 men in Taiwan. Lancet 1981; 2: 1129-1133.

11. McMahon BJ, Alberts SR, Wainwright RB, Bulkow L, Lanier AP. Hepatitis B-related sequelae. Prospective study in 1400 hepatitis B surface antigen-positive Alaska native carriers. Arch Intern Med 1990; 150: 1051-1054.
12. Sherman M, Peltekian KM, Lee C. Screening for hepatocellular carcinoma in chronic carriers of hepatitis B virus: incidence and prevalence of hepatocellular carcinoma in a North American urban population. Hepatology 1995; 22: 432-438.

13. Chen CJ, Yang HI, Su J, et al; REVEAL-HBV Study Group. Risk of hepatocellular carcinoma across a biological gradient of serum hepatitis B virus DNA level. JAMA 2006; 295: 65-73.

14. White DL, Kanwal F, El-Serag HB. Association between nonalcoholic fatty liver disease and risk for hepatocellular cancer, based on systematic review. Clin Gastroenterol Hepatol 2012; 10: 1342-1359.e2.

15. Bruix J, Sherman M; American Association for the Study of Liver Diseases. Management of hepatocellular carcinoma: an update. Hepatology 2011; 53: 1020-1022.

16. European Association for the Study of the Liver, European Organisation for Research and Treatment of Cancer. EASLEORTC clinical practice guidelines: management of hepatocellular carcinoma. J Hepatol 2012; 56: 908-943.

17. Martie A, Sporea I, Şirli R, Popescu A, Dănilă M. How often hepatocellular carcinoma has a typical pattern in contrast enhanced ultrasound? Maedica (Buchar) 2012; 7: 236240 .

18. Sporea I, Badea R, Popescu A, et al. Contrast-Enhanced U1trasound (CEUS) for the evaluation of focal liver lesions - a prospective multicenter study of its usefulness in clinical practice. Ultraschall Med 2014 Feb 21.

19. Sporea I, Martie A, Bota S, Șirli R, Popescu A, Dănilă M. Characterization of focal liver lesions using contrast enhanced ultrasound as a first line method: a large monocentric experience. J Gastrointestin Liver Dis 2014; 23: 57-63.

20. Kubota K, Ina H, Okada Y, Irie T. Growth rate of primary single hepatocellular carcinoma: determining optimal screening interval with contrast enhanced computed tomography. Dig Dis Sci 2003; 48: 581-586.

21. Zhang BH, Yang BH, Tang ZY. Randomized controlled trial of screening for hepatocellular carcinoma. J Cancer Res Clin Oncol 2004; 130: 417-422.

22. Sigal A, Volk ML, Waljee A, et al. Meta-analysis: surveillance with ultrasound for early-stage hepatocellular carcinoma in patients with cirrhosis. Aliment Pharmacol Ther 2009; 30: 37-47.

23. Trinchet JC, Chaffaut C, Bourcier V, et al; Groupe d'Etude et de Traitement du Carcinome Hépatocellulaire (GRETCH). Ultrasonographic surveillance of hepatocellular carcinoma in cirrhosis: a randomized trial comparing 3- and 6-month periodicities. Hepatology 2011; 54: 19871997.

24. Singal AG, Conjeevaram HS, Volk ML, et al. Effectiveness of hepatocellular carcinoma surveillance in patients with cirrhosis. Cancer Epidemiol Biomarkers Prev 2012; 21: 793-799.

25. Andersson KL, Salomon JA, Goldie SJ, Chung RT. Cost effectiveness of alternative surveillance strategies for hepatocellular carcinoma in patients with cirrhosis. Clin Gastroenterol Hepatol 2008; 6: 1418-1424. 
26. Gupta S, Bent S, Kohlwes J. Test characteristics of alphafetoprotein for detecting hepatocellular carcinoma in patients with hepatitis C. A systematic review and critical analysis. Ann Intern Med 2003; 139: 46-50.

27. Sherman M. Current status of alpha-fetoprotein testing. Gastroenterol Hepatol (N Y) 2011; 7: 113-114.

28. Giannini EG, Marenco S, Borgonovo G, et al; Italian Liver
Cancer (ITA.LI.CA) group. Alpha-fetoprotein has no prognostic role in small hepatocellular carcinoma identified during surveillance in compensated cirrhosis. Hepatology 2012; 56: 1371-1379.

29. Dănilă M, Sporea I, Popescu A, Sirli R, Sendroiu M. The value of contrast enhanced ultrasound in the evaluation of the nature of portal vein thrombosis. Med Ultrason 2011; 13: 102-107. 